\title{
SALÁRIOS E TECNOLOGIA NUM MODELO DE CRESCIMENTO COM RESTRIÇÃO EXTERNA*
}

\author{
Marcus Dutra
}

Gabriel Porcile

\author{
Antonio J. A. Meirelles ${ }^{* * *}$
}

RESUMO O modelo proposto formaliza uma preocupação que se encontra cada vez com mais freqüência na literatura, a saber, a de que trabalhadores que não têm acesso a condições adequadas de capacitação, saúde e motivação tendem a aprender menos, reduzindo a velocidade de inovação em produtos e processos na firma. $\mathrm{Na}$ medida em que a competitividade internacional repousa crescentemente na inovação e/ou na imitação rápida de tecnologia, um nível baixo de desenvolvimento humano implicará oportunidades de crescimento perdidas. Assim, o modelo assume que, até certo valor crítico do salário real, aumentos de salário real produzem aumentos de competitividade e da taxa de crescimento com equilíbrio externo, tornando compatíveis o crescimento econômico e a distribuição da renda, inclusive num contexto de abertura e de intensa concorrência internacional.

Palavras-chave: crescimento econômico; competitividade internacional; tecnologia e comércio internacional

Código JEL: F43 - Economic Growth of Open Economies

* Artigo recebido em 26 de janeiro de 2005 e aprovado em 25 de novembro de 2005.

** Petrobras, e-mail: marcus.dutra@bol.com

*** Professor do Departamento de Economia da Universidade Federal do Paraná (UFPR) e pesquisador do CNPq, e-mail: porcile@ufpr.br

$\overline{\star * * *}$ Professor da Faculdade de Engenharia de Alimentos da Universidade Estadual de Campinas (Unicamp) e pesquisador do CNPq, e-mail: tomze@fea.unicamp.br 


\title{
WAGES AND TECHNOLOGY IN A GROWTH MODEL WITH EXTERNAL CONSTRAINTS
}

\begin{abstract}
The model formalizes a topic that the economic literature addresses with increasing frequency, namely that workers who have no access to adequate levels of education, health and motivation tend to learn more slowly and this in turn reduces the rate of innovation in products and processes in the firm. To the extent that international competitiveness increasingly relies on innovation and imitation of technology, a low level of human development will render lost opportunities for growth. Thus, the model assumes that - up to a certain critical level of the real wage - increases in real wages lead to a higher rate of growth consistent with balance-of-payments equilibrium, which makes compatible growth and income distribution even in contexts of external openness and intense international competition.
\end{abstract}

Key words: economic growth; international competitiveness; technology and international trade 


\section{INTRODUÇÃO}

Assume-se convencionalmente que a relação entre salários reais e competitividade internacional é negativa. $\mathrm{O}$ suposto fundamenta-se em que o salário real é parte do custo unitário de produção, e este por sua vez é um indicador da capacidade das firmas de um país de competir internacionalmente. Mas, em certos casos, salários reais mais elevados podem ter conseqüências positivas sobre a competitividade em países em que os salários reais são muito baixos. Crescentemente, a literatura aponta diversos mecanismos pelos quais esses efeitos positivos poderiam acontecer.

Em primeiro lugar, em países com salário real muito baixo, a capacidade do trabalhador é negativamente afetada. A produtividade do trabalhador está positivamente associada com seu nível de consumo (Basu, 1984: 96; Bliss e Stern, 1978). Os trabalhadores desses países estão mais predispostos a ter problemas de saúde (inclusive por deficiências alimentares na gestação e nos primeiros anos da infância, que persistem posteriormente) e teriam também menor acesso à educação e a bens culturais, o que reduz sua capacidade de aprendizado. ${ }^{1}$ Em segundo lugar, a literatura sobre salários de eficiência tende a destacar que salários reais mais altos permitem que o trabalhador tenha uma motivação maior e dedique um esforço maior ao trabalho (Shapiro e Stiglitz, 1989; Ros, 2000: 320). Como resultado, o esforço que o trabalhador dedica ao aprendizado de suas tarefas, e eventualmente a melhorar o processo de trabalho, tende a aumentar com o salário real, dentro de um leque limitado de variação do salário real.

Assim, tanto pelo fator capacidade quanto pelo fator motivação, salários reais mais altos poderiam estar associados a uma taxa mais alta de aprendizado em países em desenvolvimento. Isso leva ao segundo ponto do argumento deste trabalho: competitividade e aprendizado estão positivamente associados, na medida em que cada vez mais o sucesso competitivo depende da inovação ou da imitação criativa dos inovadores, isto é, do aprendizado de novas tecnologias e de novos processos produtivos. A capacidade de se adaptar a um ambiente em permanente mudança, e de receber e processar informação de distintas fontes (inclusive de centros de pesquisa e de universidades) para entrar em mercados mais sofisticados ou em mais rápido crescimento, é um componente-chave da chamada competitividade estrutural ou sistêmica (Cimoli, 1988; Mytelka, 1999). Tudo isso reforça a idéia de que 
os impactos das variações de salários devem ser estudados considerando seus eventuais efeitos sobre a taxa de aprendizado tecnológico. Se essa taxa aumenta com o salário real, existe um espaço para que a distribuição da renda e o crescimento avancem juntos, pari passu com o aumento da competitividade internacional.

A contribuição deste trabalho é mostrar como essa relação entre salários e aprendizado pode ser tratada no marco de um modelo de crescimento com restrição em balança de pagamentos. $O$ trabalho consta de três seções, além da introdução e das conclusões.

A seção 1 apresenta as principais equações do modelo. Parte-se de uma equação de crescimento de longo prazo com equilíbrio externo, na qual a taxa de crescimento da economia depende da variação no tempo de competitividade internacional dessa economia. A variação da competitividade internacional, por sua vez, é função da taxa relativa de inovação no Norte (países líderes do ponto de vista tecnológico, que expandem a fronteira do conhecimento) e de aprendizado no Sul (formado por países que não alcançaram a fronteira tecnológica internacional).

Seguindo os modelos de catching-up tecnológico, assume-se que a taxa de aprendizado relativo depende do hiato tecnológico Norte-Sul, mas também do salário real no Sul, via os mencionados efeitos sobre a capacidade e a motivação do trabalhador. Finalmente, a variação dos salários reais no tempo é uma função do comportamento do mercado de trabalho, supondo que a oferta de trabalho cresce a uma taxa constante exógena e que a demanda de trabalho depende da taxa de crescimento da economia.

A seção 2 analisa a dinâmica do sistema e a estabilidade dos equilíbrios. A seção 3 discute o efeito, sobre os valores de equilíbrio da competitividade e do salário real, de mudanças nos parâmetros do sistema induzidas pela política econômica. O modelo mostra que uma política favorável ao aumento dos salários reais, quando se parte de níveis iniciais muito baixos, pode favorecer o aprendizado e, a partir dele, a competitividade e o crescimento, dando lugar a um círculo virtuoso por meio do qual crescimento e distribuição da renda se reforçam mutuamente. 


\section{O MODELO}

\subsection{Crescimento econômico e competitividade}

Toma-se como ponto de partida o modelo de Amable (1994), o qual introduz uma mudança no modelo de crescimento com restrição do balanço de pagamentos proposto por McCombie e Thirlwall (1994). Essa mudança consiste em incluir a variável qualidade no argumento da função de demanda de exportações, enquanto a função de demanda de importações é igual à usada por McCombie e Thirlwall. Segundo Amable, a conta corrente de um certo país dependerá de sua taxa de câmbio real, de seu nível de renda, da renda do resto do mundo e da qualidade de suas exportações. Formalmente:

$$
\begin{aligned}
& M=A\left(\frac{P^{*}}{P} E\right)^{\psi} Y^{\pi} \\
& X=B\left(\frac{P}{P^{*} E}\right)^{\eta} Z^{\varepsilon} \Omega^{\lambda}
\end{aligned}
$$

onde $M$ são as importações do Sul, $X$ são as exportações do Sul, $Z$ é a renda do Norte, $Y$ é a renda do Sul, $P$ é o nível de preços no Sul, $P^{*}$ é o nível de preços no Norte, $\Omega$ é um indicador de qualidade das exportações, $E$ é a taxa nominal de câmbio (preço em unidades monetárias domésticas de uma unidade monetária estrangeira), $\psi$ é a elasticidade preço das importações, $\pi$ é a elasticidade renda das importações, $\eta$ é a elasticidade preço das exportações, $\varepsilon$ é a elasticidade renda das exportações, $\lambda$ é a elasticidade qualidade das exportações, $A$ e $B$ são constantes positivas. As elasticidades preço são negativas, e as elasticidades com relação à renda e à qualidade são positivas. A elasticidade em qualidade assim definida é parte do que McCombie e Thirlwall (1994, cap. 3) chamam de non-price competitiveness.

Para que exista equilíbrio na conta corrente, é necessário que a seguinte condição seja satisfeita:

$$
E P^{*} M=P X
$$

ou então

$$
E P^{*} A=\left(\frac{P^{*}}{P} E\right)^{\psi} Y^{\pi}=P B\left(\frac{P}{P^{*} E}\right)^{\eta} Z^{\varepsilon} \Omega^{\lambda}
$$


Aplicando logaritmos à igualdade anterior e diferenciando com relação ao tempo, obtém-se o seguinte resultado:

$$
e+p^{*}+\psi\left(p^{*}+e-p\right)+\pi y=\eta\left(p-p^{*}-e\right)+p+\varepsilon Z+\lambda \omega
$$

onde as letras minúsculas representam as taxas de variação das variáveis no tempo (por exemplo, $p=\frac{\partial p / \partial t}{p}$ ).

Da equação (5) obtemos a taxa de crescimento de longo prazo da economia compatível com o equilíbrio na conta corrente:

$$
\begin{aligned}
& y=\frac{1}{\pi}\left(\eta\left(p-p^{*}-e\right)+\psi\left(p-p^{*}-e\right)+\left(p-p^{*}-e\right)+\varepsilon z+\lambda \omega\right) \\
& y=\frac{1}{\pi}\left((\eta+\psi+1)\left(p-p^{*}-e\right)+\varepsilon z+\lambda \omega\right)
\end{aligned}
$$

É fácil ver que a taxa de crescimento de longo prazo com equilíbrio em conta corrente depende da variação da taxa de câmbio real, da variação da renda do resto do mundo e da variação da qualidade das exportações. É o resultado conhecido como Lei de Thirwall, com a inclusão de um novo componente, a saber, a variação da qualidade das exportações no tempo.

Para tornar o modelo mais operacional e estudar suas conexões com tecnologia e salários, adotam-se alguns supostos simplificadores. O primeiro suposto é que a variação da qualidade é função do nível das assimetrias tecnológicas internacionais. ${ }^{2}$ Mais especificamente, assume-se que a variação da competitividade em qualidade é uma função inversa do hiato tecnológico Norte-Sul, dada por $S=(T s / T n)$, onde $T s$ e $T n$ denotam o estoque de conhecimentos tecnológicos no Sul e no Norte, respectivamente. Formalmente:

$$
\omega=S=\left(\frac{T_{S}}{T_{N}}\right)
$$

O segundo suposto, de uso freqüente neste tipo de modelo, consiste em supor que o princípio da paridade do poder de compra se verifica no longo prazo (i.e., $p-p^{*}-e=0$ ). O terceiro suposto é que a economia opera em re- 
gime de taxa de câmbio fixa, portanto $e=0$. A combinação dos dois supostos anteriores implica a plena convergência entre a taxa de inflação interna e a taxa de inflação externa $\left(p=p^{*}\right)$.

Usando os supostos anteriores, tem-se que:

$$
y=\frac{1}{\pi}(\varepsilon z+\lambda \omega)=\frac{1}{\pi}(\varepsilon z+\lambda S)
$$

Assim, a taxa de crescimento da renda dos países menos desenvolvidos será inversamente proporcional à elasticidade renda das importações $(\pi)$ e diretamente proporcional à taxas de crescimento da renda do mundo $(z)$, à função inversa do hiato tecnológico $(S)$ e às elasticidades renda $(\varepsilon)$ e de qualidade $(\lambda)$ das exportações.

$S$ varia no tempo como função do aprendizado no Sul, dada uma taxa constante de progresso técnico no Norte. Na próxima seção discutem-se os determinantes do aprendizado no Sul: o hiato tecnológico Norte-Sul, que define o potencial de imitação existente para o Sul, e a capacidade dos trabalhadores do Sul de absorver e desenvolver a tecnologia estrangeira, que depende do salário real.

\subsection{Aprendizado}

A evolução da variável $S$ no tempo depende do hiato tecnológico e do salário real no Sul. A influência do hiato é um aspecto bem documentado na literatura sobre catching-up tecnológico, como em Fagerberg $(1988,1995)$. A idéia é que quanto maior for o hiato tecnológico, maiores serão as oportunidades de imitação, maior a difusão de tecnologia e mais alta a taxa de aprendizado no Sul. Como $S$ é o inverso do hiato tecnológico, a velocidade de aprendizado cresce com o aumento desse hiato.

Com relação ao salário real, assume-se que a taxa de aprendizado aumenta com o salário real até um certo valor crítico, a partir do qual aumentos do salário real já não afetam o aprendizado. O suposto é razoável, na medida em que o efeito negativo dos baixos salários tem a ver com deficiências na saúde e na formação do trabalhador. Quando essas deficiências são atendidas, o trabalhador pode alcançar seu potencial, independentemente de novos aumentos do salário real. Formalmente: 


$$
\mid \begin{array}{lrl}
s=a_{0} & +a_{1} W-a_{2} S & \text { para } W \leq W c \\
s=a_{0}+a_{1} W_{c} & -a_{2} S & \text { para } W>W c
\end{array}
$$

onde $s=\frac{d \ln S}{d t}$ é a taxa de crescimento da inversa do hiato, $W$ é o salário real do Sul e Wc é o valor crítico (que se supõe exógeno) do salário real a partir do qual já não afeta a taxa de aprendizado no Sul. Todos os parâmetros são positivos. O parâmetro $a_{1}$ é uma medida do efeito do salário real sobre o aprendizado. $\mathrm{O}$ parâmetro $a_{2}$ indica a velocidade com que se perdem as oportunidades de imitação quando o hiato se reduz. Quanto maior $a_{2}$, menor é a capacidade economia de aproveitar a existência de um hiato tecnológico para promover o seu próprio processo de aprendizado.

\subsection{O Mercado de trabalho}

A variação do salário relativo do Sul é uma função (a) da diferença na taxa de aumento da oferta e demanda de trabalho e (b) do conflito entre empresários e sindicatos quando os empresários são price-takers (e, em conseqüência, o mark-up é flexível: deve-se lembrar que se supõe a plena convergência dos preços externos e internos). A demanda de trabalho depende, por um lado, da taxa de crescimento da economia e, portanto, da sua competitividade em qualidade (equação 9). Supondo dado o aumento da oferta de trabalho no tempo, quanto maior o aumento da demanda de trabalhadores, maior tenderá a ser a variação do salário relativo. Mas o conflito entre capital e trabalho também afeta a evolução dos salários reais. À medida que o salário real é mais alto e a margem de lucros se reduz, aumenta a resistência empresarial a outorgar novos aumentos de salários (Carlin e Soskice, 1990: 140 - 143). Assim, para uma mesma variação no nível de emprego, a variação do salário real será menor quando o nível do salário real for maior.

Em resumo, a variação percentual do salário real no tempo, denotada como $w=[d(\ln W) / d t)$, é inversamente proporcional ao nível do salário real e diretamente proporcional à competitividade do Sul. Formalmente:

$$
w=b_{o}-b_{1} W+b_{2} S
$$

onde todos os parâmetros são positivos; $b_{1}$ depende da capacidade dos empresários (trabalhadores) de evitar (promover) cortes na margem de lucro; 
$b_{2}$ depende do impacto do crescimento sobre a variação do emprego, afetando a barganha salarial.

\section{DINÂMICA E ESTABILIDADE}

\subsection{Equilíbrio}

A discussão anterior define um sistema de duas equações diferenciais, uma das quais exprime o comportamento do mercado de trabalho e a outra a dinâmica tecnológica (e de competitividade em qualidade). Com efeito, tem-se que:

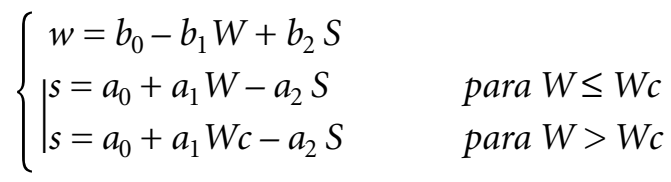

A equação (13) pode ser divida em dois subsistemas:

$$
\begin{array}{ll}
W=[0, W c) & W=[W c, \infty) \\
\left\{\begin{array}{l}
w=b_{0}-b_{1} W+b_{2} S \\
s=a_{0}-a_{1} W-a_{2} S
\end{array} \quad(14)\right. & \left\{\begin{array}{l}
w=b_{0}-b_{1} W+b_{2} S \\
s=a_{0}-a_{1} W c-a_{2} S
\end{array}\right.
\end{array}
$$

E, reordenando os termos, tem-se:

$$
\left\{\begin{array} { l } 
{ w + b _ { 1 } W - b _ { 2 } S = b _ { 0 } } \\
{ s = a _ { 1 } W + a _ { 2 } S = a _ { 0 } }
\end{array} \quad \left\{\begin{array}{l}
w+b_{1} W-b_{2} S=b_{0} \\
s+a_{2} S=a_{0}+a_{1} W c
\end{array}\right.\right.
$$

Em equilíbrio, $S(t)=S^{\star}, \mathrm{W}(t)=W^{\star}$ e $s=w=0$, portanto:

$$
\begin{array}{ll}
W=[0, W c) & W=[W c, \infty) \\
\left\{\begin{array} { l } 
{ + b _ { 1 } W - b _ { 2 } S = b _ { 0 } } \\
{ - a _ { 1 } W + a _ { 2 } S = a _ { 0 } }
\end{array} \quad ( 1 8 ) \quad \left\{\begin{array}{l}
+b_{1} W-b_{2} S=b_{0} \\
+a_{2} S=a_{0}+a_{1} W c
\end{array}\right.\right.
\end{array}
$$


Para $W^{\star} \leq W c$, tem-se que em equilíbrio:

$$
W^{\star}=\frac{a_{2} b_{0}+b_{2} a_{0} \mathrm{e}}{b_{1} a_{2}-b_{2} a_{1}} \text { e } S^{\star}=\frac{a_{1} b_{0}+b_{1} a_{0}}{b_{1} a_{2}-b_{2} a_{1}}
$$

Quando o equilíbrio ocorre para um salário real no Sul maior que o salário crítico, os valores de equilíbrio são dados pelas seguintes equações:

$$
W^{\star}=\frac{b_{0}}{b_{1}}+\frac{b_{2}\left(a_{0}+a_{1} W c\right)}{b_{1} a_{2}} \text { e } S^{\star}=\frac{\left(a_{0}+a_{1} W c\right)}{a_{2}}
$$

Para evitar cenários inconsistentes não apenas no ponto de equilíbrio, mas durante todo o período transiente da dinâmica econômica, são adicionadas restrições aos valores que podem assumir as variáveis. A primeira restrição impede que o Sul supere o Norte em termos tecnológicos, ou seja, $T_{S} / T_{N}$ não pode ser maior do que a unidade.

$$
T_{S} / T_{N} \leq 1
$$

Outro cenário que deve ser evitado durante a dinâmica de transição é que o lucro dos capitalistas seja negativo. Para isso, é necessário que a massa de salários seja menor que o produto (expressas ambas as variáveis em termos reais):

$$
W L<Y
$$

onde $W$ é o salário real; $L$ é o nível de emprego; $Y$ é a renda do Sul. Se a produtividade do trabalho é $A=Y / L$, e assume-se que ela cresce linearmente com a inversa do hiato tecnológico, $A=K S$, a restrição (21) pode ser escrita como:

$$
W<K S
$$

A equação (22) é imediatamente intuitiva, já que apenas implica que o salário real não pode ser maior do que a produtividade física do trabalho.

As figuras 1A e 1B mostram graficamente os pontos de equilíbrio do sistema. As áreas tracejadas são aquelas inconsistentes com (20) ou (22).

\subsection{Estabilidade}

A seguir, analisar-se-á a estabilidade dos pontos fixos a partir da matriz jacobiana dos subsistemas de equações diferenciais anteriormente apresentados. Os subsistemas (14) e (15) podem ser escritos como: 
Figuras $1 A$ e 1B: Equilíbrio do salário e do hiato tecnológico
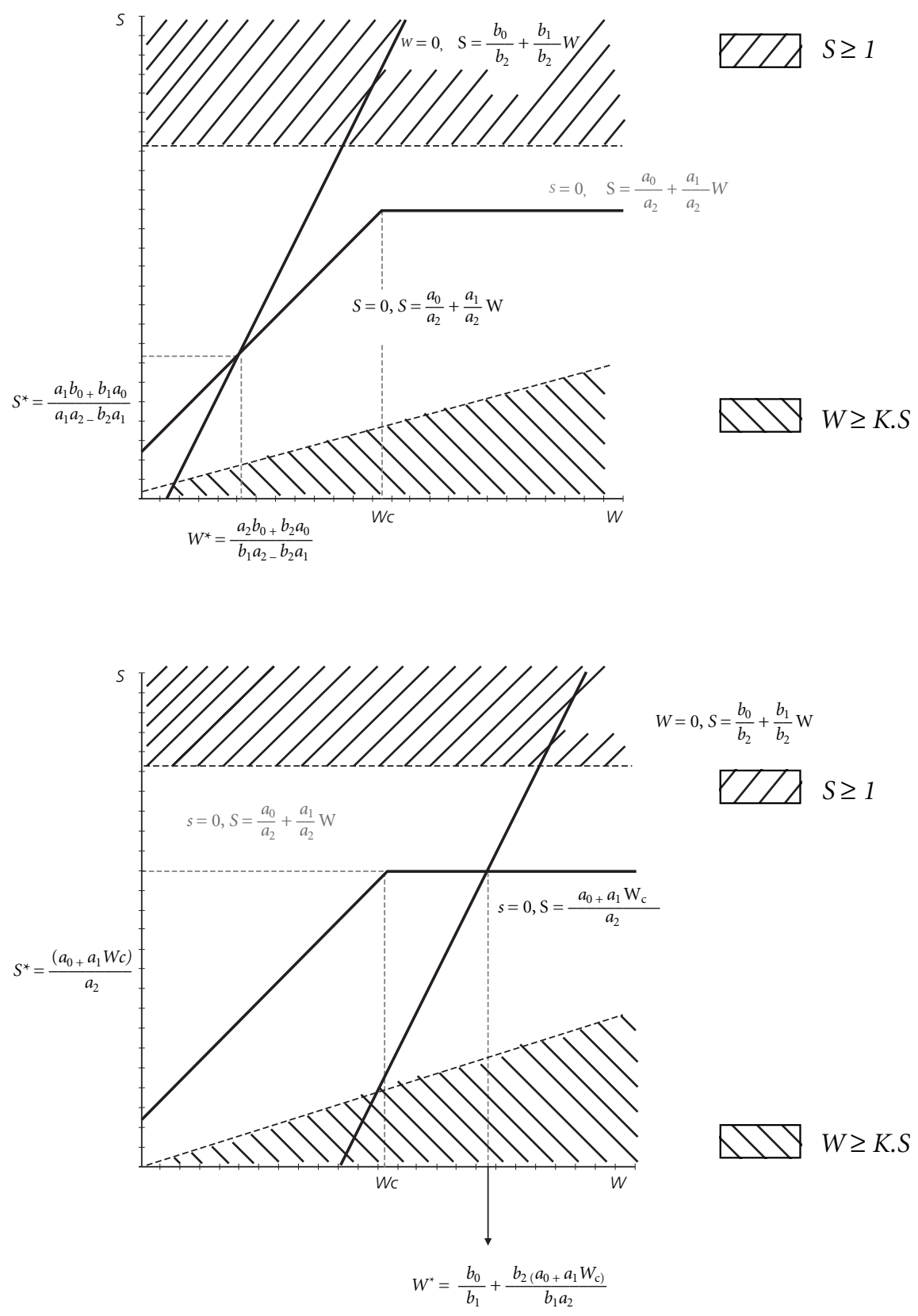


$$
\begin{aligned}
& {\left[\begin{array}{l}
w \\
s
\end{array}\right]=\left[\begin{array}{cc}
-b_{1} & b_{2} \\
a_{1}-a_{2}
\end{array}\right]+\left[\begin{array}{l}
W \\
S
\end{array}\right]+\left[\begin{array}{l}
b_{0} \\
a_{0}
\end{array}\right]} \\
& {\left[\begin{array}{l}
w \\
s
\end{array}\right]=\left[\begin{array}{cc}
-b_{1} & b_{2} \\
0-a_{2}
\end{array}\right]\left[\begin{array}{l}
W \\
S
\end{array}\right]+\left[\begin{array}{c}
b_{0} \\
a_{0}+a_{1} \mathrm{Wc}
\end{array}\right]}
\end{aligned}
$$

Onde

$$
J_{w \leq w c}=\left[\begin{array}{cc}
-b_{1} & b_{2} \\
a_{1} & -a_{2}
\end{array}\right] \text { e } J_{w>w^{c}}=\left[\begin{array}{cc}
-b_{1} & b_{2} \\
-a_{2} & 0
\end{array}\right]
$$

são as matrizes jacobianas desses subsistemas. Como se sabe, a análise de estabilidade pode ser feita a partir dessas matrizes. Analisar-se-á primeiramente o caso em que $W^{\star} \leq W c$. $\mathrm{O}$ traço e o determinante do primeiro subsistema são os seguintes:

$$
\operatorname{tr} J=-b_{1}-a_{2} \quad(25), \quad|J|=b_{1} a_{2}-b_{2} a_{1}
$$

Como todos os parâmetros $a_{i}$ e $b_{i}$ são positivos, sabe-se, sem ambigüidade, que para $W^{\star} \leq W c$, tem-se que $\operatorname{tr} J<0$. Mas o sinal do determinante não é evidente. Há, entretanto, algumas condições que devem ser satisfeitas para que a interseção das curvas $s=0$ e $w=0$ ocorra em um ponto no qual $0<W^{\star} \leq W c$. Essas condições serão bastante úteis na análise do sinal de $|J|$. A equação que descreve a competitividade em função do nível salarial a partir de $w=0$ possui um coeficiente linear negativo igual a $-b_{0} / b_{2}$. Por sua vez, a isolinha $s=0$ tem coeficiente linear positivo $a_{0} / a_{2}$. Portanto, para que as retas se interceptem no primeiro quadrante, é necessário que o coeficiente angular da reta $s=0$ seja menor que o da reta $w=0$. Sendo assim,

$$
a_{1} / a_{2}<b_{1} / b_{2}
$$

Essa condição é necessária, embora não seja suficiente, para garantir que $0<W^{\star} \leq W c$. Do ponto de vista da análise de estabilidade, a verificação dessa condição é suficiente para que o equilíbrio seja estável, pois dela conclui-se que $b_{1} a_{2}>b_{2} a_{1}$ e, portanto, $|J|>0$. Dessa forma, se $(\operatorname{tr} J)^{2} \geq 4|J|$, o ponto de equilíbrio será um nó estável, e se $(\operatorname{tr} J)^{2}<4|J|$, o equilíbrio será um foco estável. ${ }^{3}$ 
Figuras 2A e 2B: Campos vetoriais de salário e hiato tecnológico
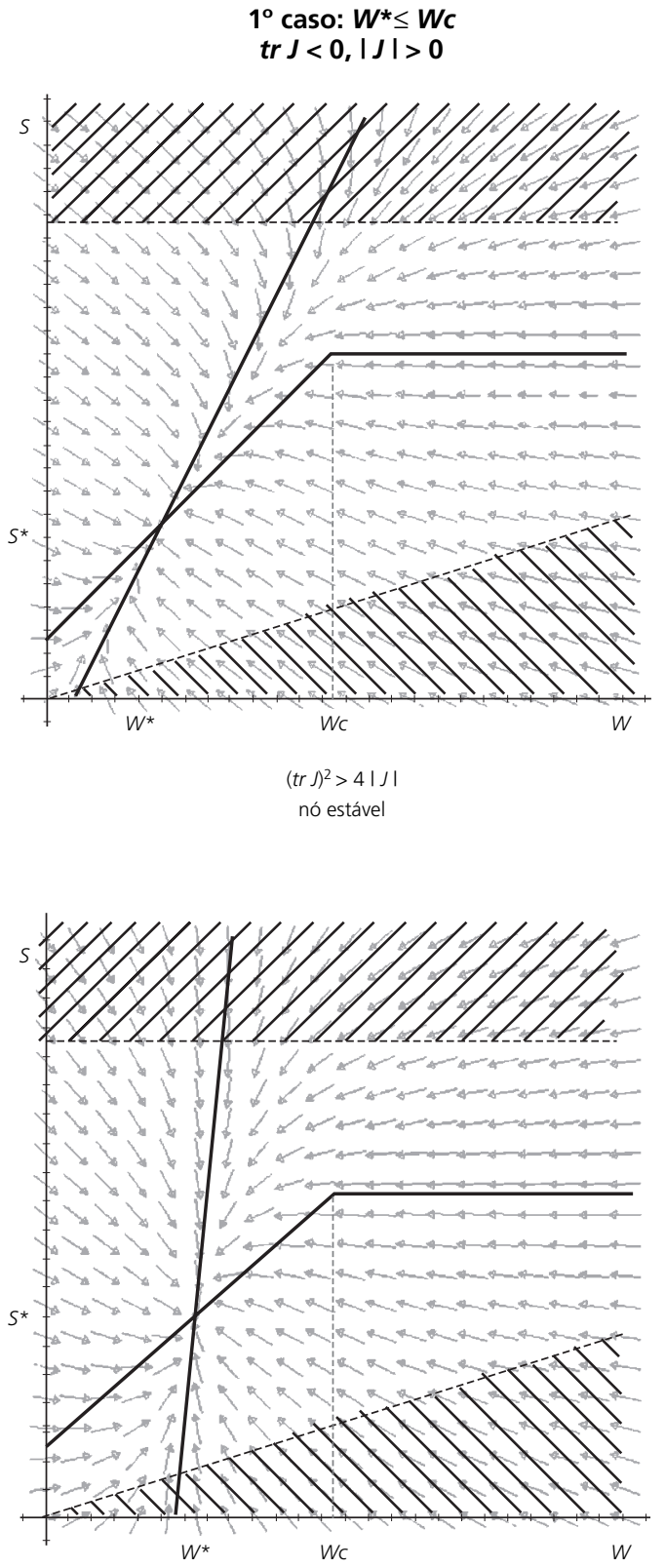

$\left(\operatorname{tr} \int\right)^{2}<4|\jmath|$

foco estável 


\section{$2^{\circ}$ caso: $W^{*}>W c$ \\ $\operatorname{tr} J<0,|J|>0$}
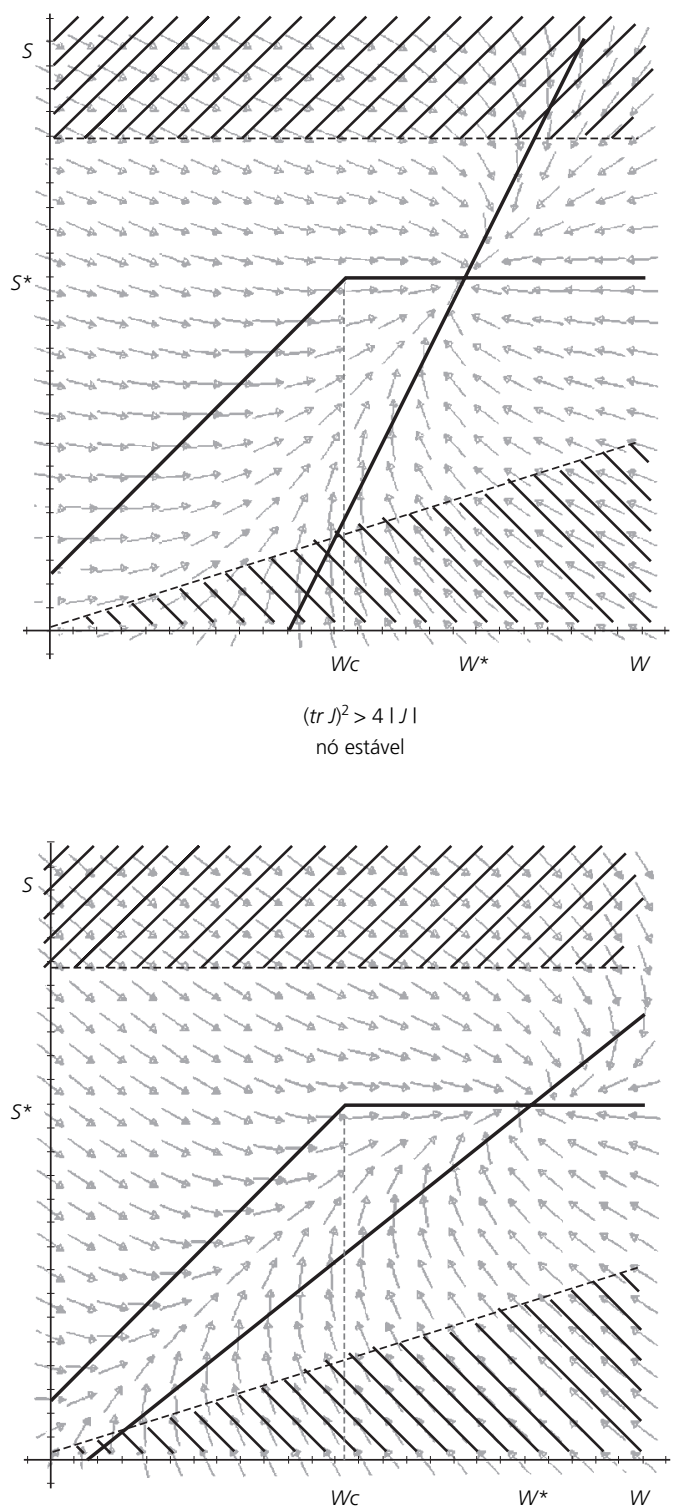

$\left(\operatorname{tr} \int\right)^{2}<4|\jmath|$

foco estável 
No caso em que $W^{*}>W c$, temos os seguintes resultados no cálculo do traço e do determinante:

$$
\operatorname{tr} J=-b_{1}-a_{2} \quad(28), \quad|J|=b_{1} a_{2}
$$

O traço e o determinante da jacobiana são, respectivamente, negativo e positivo para quaisquer valores dos parâmetros $b_{1}$ e $a_{2}$. Assim, se $(\operatorname{tr} J)^{2} \geq 4$ $|J|$, o ponto de equilíbrio será um nó estável, e se $(\operatorname{tr} J)^{2}<4|J|$, o ponto de equilíbrio será um foco estável.

Em resumo, os pontos de equilíbrio possíveis do sistema são estáveis. As figuras $2 A$ e $2 B$ mostram os campos vetoriais de $W$ e $S$, traçados a partir das equações $s=s(S, W)$ e $w=w(S, W)$.

\section{ALGUMAS IMPLICAÇÕES DO MODELO}

Uma primeira conclusão que surge do modelo é que se o equilíbrio se alcança antes de $W c$, esse equilíbrio será ineficiente, dado que para uma economia é preferível ter melhores salários e maior crescimento ao mesmo tempo. Com efeito, quaisquer pares de valores $\left(W^{\star}, \mathrm{S}^{\star}\right)$, em que $W^{\star}<W c$ implicam que a economia não está explorando plenamente as complementaridades existentes entre crescimento e salários reais, aceitando, simultaneamente, um nível mais baixo de salários e uma taxa menor de crescimento do que em quaisquer configurações alternativas a partir de $W c$.

Que é necessário para que $W^{\star}$ supere $W c$ ? O equilíbrio ocorrerá após o ponto crítico $W c$ se, por exemplo, $b_{1} a_{2}<b_{2} a_{1}$ (obtida a partir da equação 27). Para que isso aconteça, a interação positiva entre salários e competitividade (salário maior eleva a competitividade, que por sua vez eleva os salários) deverá superar a interação negativa entre a redução do aprendizado (e do aumento da competitividade), dada pelo fechamento do hiato, e a crescente luta distributiva entre o capital e o trabalho. Essa interação negativa limita os aumentos salariais quando o salário real se eleva. Neste caso, políticas que fortaleçam a capacidade negociadora dos trabalhadores e políticas orientadas a elevar a capacitação do trabalhador seriam capazes de promover, simultaneamente, uma competitividade mais alta e uma melhor distribuição da renda. O primeiro tipo de política seria captado no modelo via redução no coeficiente $b_{1}$, enquanto o segundo poderia ser captado via redução do coeficiente $a_{2}$, indicando elevada capacidade de aproveitar no Sul as oportunidades de imitação. 
Esse resultado do modelo tende a ser coincidente com os estudos que mostram que um padrão muito desigual de distribuição tende a estar associado com uma taxa menor de inovação e de crescimento (Engerman e Sokoloff, 1994; Aghion e Williamson, 2000). Uma economia na qual as políticas tivessem um viés claramente negativo com relação à distribuição de renda (por exemplo, por que as políticas públicas são cooptadas pelos setores mais afluentes, ou por que existe uma grande repressão das demandas dos setores mais pobres) teria maiores probabilidades de se encontrar num equilíbrio aquém de $W c$.

O mecanismo aqui proposto de reforço entre crescimento, competitividade e salários reais numa economia aberta sujeita à restrição de balanço de pagamentos complementa o que fora encontrado por Blecker (1989). No caso desse autor, essa complementaridade dava-se por meio das elasticidades preço da demanda de exportações e importações. Uma queda no markup e a conseqüente melhora na distribuição da renda de um país estariam associadas a maiores competitividade e crescimento, no modelo de Blecker, apenas se as elasticidades preço das exportações e das importações são altas o suficiente em relação à elasticidade renda das importações. Caso contrário, o aumento no nível de atividade provocado pela melhor distribuição geraria um desequilíbrio externo capaz de frustrar o crescimento (Blecker, 1989: 403). No modelo aqui apresentado, esse tipo de efeito está excluído porque se assumiu perfeita convergência entre os preços internos e externos. No entanto, o efeito benéfico sobre a competitividade do aumento do salário real pode efetivamente ocorrer a partir do aumento da taxa de aprendizado: a conexão entre crescimento e distribuição é dada pelo aprendizado tecnológico, que aumenta a competitividade em qualidade do Sul.

A situação é um pouco diferente quando o equilíbrio acontece além do ponto crítico do salário real (ver tabela 1 , que resume os efeitos das mudanças dos parâmetros do modelo sobre os valores de equilíbrio do salário real e da inversa do hiato). Aumentos salariais muito intensos poderiam levar a uma situação de esmagamento de lucros, um cenário excluído do modelo ex-hipotese. Mas, também neste caso, uma política destinada a elevar o coeficiente de aprendizado do trabalhador contribuiria para alcançar valores mais altos de $S^{\star}$ e $W^{\star}$. Da mesma forma, políticas que procurem maximizar a imitação de tecnologia (menor $a_{2}$ ) também contribuem para aumentar os valores do salário real e a competitividade de equilíbrio. 
Tabela 1: Efeitos das mudanças nos parâmetros do sistema sobre os valores de $W^{*}$ e $S$ *

\begin{tabular}{ccc}
\hline Parâmetro & Efeito sobre $W^{*}$ & Efeito sobre $S^{*}$ \\
\hline $\mathrm{a}_{0}$ & $(+)$ & $(+)$ \\
\hline $\mathrm{a}_{1}$ & $(+)$ & $(+)$ \\
\hline $\mathrm{a}_{2}$ & $(-)$ & $(-)$ \\
\hline $\mathrm{b}_{0}$ & $(+)$ & $(+)$ \\
\hline $\mathrm{b}_{1}$ & $(-)$ & $(-)$ \\
\hline $\mathrm{b}_{2}$ & $(+)$ & $(+)$ \\
\hline
\end{tabular}

\begin{tabular}{ccc}
\hline Parâmetro & Efeito sobre $W^{*}$ & Efeito sobre $S^{*}$ \\
\hline$a_{0}$ & $(+)$ & $(+)$ \\
\hline$a_{1}$ & $(+)$ & $(+)$ \\
\hline$a_{2}$ & $(-)$ & $(-)$ \\
\hline$b_{0}$ & $(+)$ & $(0)$ \\
\hline$b_{1}$ & $(-)$ & $(0)$ \\
\hline$b_{2}$ & $(+)$ & $(0)$ \\
\hline
\end{tabular}

É importante observar que quanto maior $W c$, maiores serão os valores de $S^{\star}$ e $W^{\star}$ do segundo equilíbrio. $W c$ foi suposto constante no modelo, mas parece razoável admitir que ele tende a aumentar à medida que a economia se torna mais sofisticada e competitiva em bens e serviços mais intensivos em conhecimentos. O Wc poderia ser relativamente baixo num país que produz aço e sapatos baratos, mas pode ser muito alto para um país que produz aços especiais e sapatos intensivos em design. Em outras palavras, a própria transformação da economia redefine o que pode ser considerado um trabalhador plenamente capacitado e motivado. Assim, Wc seria uma espécie de alvo em movimento, que não poderia ser alcançado com um único tipo de política, de uma vez e para sempre.

\section{COMENTÁRIOS FINAIS}

O modelo proposto formaliza, no contexto dos modelos de crescimento com restrição externa, uma preocupação que se encontra cada vez com mais freqüência na literatura, a saber, a de que trabalhadores que não têm acesso a condições adequadas de capacitação, saúde e motivação tendem a aprender menos, reduzindo a velocidade da mudança de produtos e processos. $\mathrm{Na}$ medida em que a competitividade internacional repousa crescentemente na inovação e/ou na imitação rápida de tecnologia, um nível baixo de desenvolvimento humano implicará oportunidades de crescimento perdidas. 
Admite-se que o aprendizado não aumenta indefinidamente com o salário real, mas apenas até um valor crítico do salário igual a Wc. Uma economia somente estaria aproveitando de forma eficiente as complementaridades entre competitividade e salário real se o salário superasse esse valor crítico. Quando isso acontece, o papel das políticas de elevação do salário real se torna menos central, e ganham relevância as políticas destinadas a fortalecer o aprendizado e a promover setores mais intensivos em conhecimentos.

\section{NOTAS}

1. Como observam Ranis e Stewart (2002), “un mayor desarrollo humano genera más crecimiento a medida que una población más sana y educada contribuye a mejorar el desempeño económico".

2. Sobre esse ponto, ver Verspagen (1993), cap. 5, e Canuto, Higachi e Porcile (1999).

3. De forma mais intuitiva, pode-se dizer que a estabilidade requer que o impacto negativo do salário real sobre sua própria variação $\left(b_{1}\right)$ supere o impacto positivo do salário real sobre $S\left(a_{1}\right)$, de forma a evitar um processo explosivo de aumentos dos salários reais e o correspondente aumento requerido da competitividade.

\section{REFERÊNCIAS BIBLIOGRÁFICAS}

AGHION, P., WILLIAMSON, J. (1998) Growth, Inequality and Globalization. Cambridge University Press.

AMABLE, B. "National effects of learning, international specialization and growth paths". In: D. Foray e C. Freeman (eds.), Technology and the Wealth of Nations. Londres: Pinter Publishers.

BASU, K. (1984) The Less Developed Economy: A Critique of Contemporary Theory. Oxford: Basil Blackwell.

BLECKER, R. A. "International competition, income distribution and economic growth", Cambridge Journal of Economics, n. 13, p. 395-412.

BLISS, C. L., STERN, N. (1978) "Productivity, wages and nutrition", Journal of Development Economics, n. 5 .

CANUTO, O., HIGACHI, H., PORCILE, G. (1999) "Modelos evolucionistas de crescimento endógeno", Revista de Economia Política, v. 19, n. 4, p. 53-77.

CARLIN, W., SOSKICE, D. (1990) Macroeconomics and the Wage Bargain. Oxford University Press.

CIMOLI, M. (1988) “Technological gaps and institutional asymmetries in a North-South model with a continuum of goods", Metroeconomica, n. 39, p. 245-274.

ENGERMAN, S., SOKOLOFF, K. (1997) "Factor endowments, institutions and differential paths of growth among new world economies: a view from economic historians of the 
United States". In: S. Haber (ed.), How Latin America Fell Behind. Stanford University Press, p. 260-304.

FAGERBERG, J. (1988) “International competitiveness”, Economic Journal, n. 98, p. 355-374.

(1995) "Convergence or divergence? The impact of technology on why growth rates differ”, Journal of Evolutionary Economics, n. 5, p. 269-284.

McCOMBIE, J. S. L., THIRLWALL, A. P. (1994) Economic Growth and the Balance of Payments Constraint. Londres: St Martin's Press.

MYTELKA, L. K. (1995) “Competition, innovation and competitveness: a framework for analysis”. In: L. K. Mytelka, Competion, Innovation and Competitiveness in Developing Countries. Paris: OECD.

RANIS, G., STEWART, F. (2002) “Crecimiento económico y desarrollo humano en América Latina”. Revista de la CEPAL, n. 78, dezembro, p. 8-18.

ROS, J. (2000) Development Theory and the Economics of Growth. The University of Michigan Press.

SHAPIRO, C., STIGLITZ, J. (1989) “Equilibrium Unemployment as a Worker Discipline Device”. American Economic Review, n. 74, p. 433-444.

VERSPAGEN, B. (1993) Uneven Growth Between Interdependent Economies. Londres: Avebury. 